\title{
Civilisations
}

Revue internationale d'anthropologie et de sciences

humaines

$54 \mid 2006$

Expériences de recherche en République

démocratique du Congo

\section{Une semaine d'enquêtes ethnographiques dans les commissariats de Lubumbashi}

Olivier Kahola

\section{(2) OpenEdition}

1 Journals

Édition électronique

URL : http://journals.openedition.org/civilisations/288

DOI : $10.4000 /$ civilisations. 288

ISSN : 2032-0442

Éditeur

Institut de sociologie de l'Université Libre de Bruxelles

Édition imprimée

Date de publication : 1 avril 2006

Pagination : 25-32

ISBN : 2-87263-006-6

ISSN : 0009-8140

Référence électronique

Olivier Kahola, " Une semaine d'enquêtes ethnographiques dans les commissariats de Lubumbashi », Civilisations [En ligne], 54 | 2006, mis en ligne le 01 avril 2009, consulté le 01 mai 2019. URL : http:// journals.openedition.org/civilisations/288 ; DOI : 10.4000/civilisations.288

(c) Tous droits réservés 


\title{
Une semaine d'enquêtes ethnographiques dans les commissariats de Lubumbashi
}

\author{
Olivier KAHOLA
}

Résumé : Au sein des Services spéciaux de la police de Lubumbashi, les pots-de-vin et les actes de violence sont courants. Ces pratiques participent plus largement d'un monnayage des plaintes, seule ressource des agents suite aux ruptures des paiements de l'Etat. Conduire une enquête parmi les forces de l'ordre n'est pas aisé car la présence d'un enquêteur suscite de nombreuses craintes quant au dévoilement de cet ordre des choses : pourquoi est-il là? pour qui travaille-t-il? que cherche-t-il? L'acquisition de la confiance devient l'enjeu déterminant de l'enquête, car le chercheur est d'abord confronté au mur du silence. Cet article montre comment elle peut être gagnée à travers de simples gestes de camaraderie, de complicité, d'humour partagé et de nuits de garde passées ensemble. La participation aux activités du commissariat, à travers l'enregistrement des plaintes, fut aussi un élément capital pour cette acceptation, ce qui ouvre un débat sur la déontologie dans le contexte de l'enquête.

Mots clés : observation participante, police, justice, corruption, Lubumbashi.

Summary: Bribes and violence are commonplace in a Lubumbashi police station. They are practices inseparable from the 'bidding for justice' between plaintiffs and suspects: because agents are not paid by the state, justice goes to the highest bidder. Carrying out research in the police milieu creates serious fears about making these practices known: Why is the researcher here? Who is he working for? What is he really looking for? Establishing a relationship based on trust is crucial to the outcome of such a study because the researcher will not have access to information otherwise. This article shows how trust can be established by simple acts of friendship, complicity and good humour, or a late night spent together at the station. While the participation in registering complaints was also crucial in this process, it also raises the question of research ethics.

Key words: participatory observation, police, justice, corruption, Lubumbashi. 


\section{Introduction}

Cet article a pour objet de décrire et de commenter un travail d'enquête réalisé

$\checkmark$ dans le cadre d'une étude sur la criminalité entreprise par l'Observatoire du changement urbain (OCU), une structure de recherche née de la collaboration entre l'Université de Lubumbashi et les universités francophones de Belgique. Cette étude visait deux objectifs : d'une part, établir un état des lieux de la criminalité à Lubumbashi; d'autre part, comprendre le fonctionnement des forces de l'ordre.

Pour rencontrer ces deux objectifs, il a notamment été décidé de s'intéresser au traitement des plaintes au niveau des commissariats. Les six commissariats de la ville et les Services spéciaux (dont la compétence couvre toute l'étendue urbaine et parfois davantage) sont placés sous l'autorité du commandant-ville. Les commissariats sont dirigés par un commandant de compagnie assisté d'un commandant de compagnie en second. Les Services spéciaux comprennent quatre bureaux, chacun dirigé par un commandantchef secondé par deux ou trois officiers de police judiciaire : le bureau de renseignement, qui reçoit les signalements et les informations sur les infractions; le bureau de recherche, qui recherche et appréhende les suspects; le bureau d'exploitation et d'investigation, qui instruit les dossiers; le bureau d'études, de documentation et de statistiques (Kaumba Lufunda $2004: 2$ ).

C'est dans ces Services spéciaux que j'ai été attaché du vendredi 2 au jeudi 8 août 2002 pour une semaine d'enquête. J'ai travaillé de jour comme de nuit selon la programmation des permanences ${ }^{1}$. L'expérience que je rapporte n'est autre que les faits vécus et les stratégies auxquelles j'ai dû recourir face aux difficultés rencontrées. Habitué aux enquêtes dans les ménages, j'ai pu à cette occasion mesurer combien celles-ci sont différentes de celles conduites dans les institutions : j'étais perçu ici comme un intrus, un espion, d'où l'omniprésence de la méfiance et l'obligation de développer des stratégies nouvelles.

Pour faciliter l'accès des enquêteurs aux services de police, l'OCU avait associé le commandant-ville à notre recherche : il s'agit de la plus haute autorité en place. Ce dernier, ayant compris le bien-fondé de l'étude, s'est impliqué pour faciliter les contacts entre les enquêteurs et les commandants. Il a d'ailleurs organisé une tournée avec l'équipe d'enquêteurs dans les différents commissariats et sous-commissariats. Le commandantville a expliqué à ses subalternes les visées de l'étude, tout en les exhortant à une franche collaboration avec les enquêteurs. Cette initiative a facilité l'accès aux postes de police : les commandants ne pouvaient nous interdire l'accès à leurs bureaux. Mais une fois dans le bureau, tout restait à négocier.

\section{Vendredi : premiers contacts}

Vendredi matin, le 2 août, je me présente auprès des commandants des Services spéciaux pour commencer mon enquête. J'ai senti une méfiance absolue. Ils me considèrent

1. Nous étions quatre chercheurs et nous nous relayions en respectant les heures de permanence qui étaient de 7 h30 à 21 h00 en journée et de 21 h00 à 7 h30 la nuit. 
visiblement comme un intrus et s'estiment espionnés dans leur activité professionnelle. On est très loin des conditions optimales : « il ne suffit pas de convaincre une personne de participer à la recherche et de créer un contexte qui lui permette d'être à l'aise dans la situation d'entretien. Encore faut-il qu'elle se sente assez en confiance pour accepter de vraiment parler» (Poupart $1997: 190)$.

Le premier jour est consacré à l'acquisition de cette confiance de mes « hôtes ». Il me faut les convaincre que je ne suis pas un intrus à la solde de l'autorité. Je leur explique franchement le but de l'étude en leur disant que si j'ai été introduit par le commandantville, ce n'est pas parce que je suis son agent secret mais car je ne peux pas mener cette étude dans leurs services sans en informer l'autorité compétente. Je leur signale également que nos échanges resteront anonymes et qu'ils ne doivent pas craindre d'éventuelles conséquences de leurs témoignages.

L'objet de ma recherche n'a pas été rapidement compris lors de ces enquêtes. Je pense ici à un commandant à qui j'ai expliqué à maintes reprises le but de l'étude : il ne comprenait malheureusement pas. Pour garder sa crédibilité, il a feint de comprendre et m'a dit qu'il était disposé pour l'entretien. Cependant, lorsque que je lui posais des questions, il me paraissait fort réservé et expédiait l'entretien. J'ai vite compris qu'il fallait arrêter là. Je l'ai remercié en lui annonçant que je reviendrais le lendemain. Ce jour-là, il m'a à nouveau demandé de lui expliquer les visées de l'enquête. Ma constance a fini par le convaincre et il devint plus ouvert que le jour précédent.

Ce vendredi soir, je dois rester avec un commandant. Mais celui-ci, par méfiance envers moi sans doute, me demande d'aller plutôt veiller au corps de garde avec les policiers assurant la sécurité des détenus. Je lui explique que c'est avec lui que je dois normalement travailler, mais il rétorque : «non, je suis assermenté et je ne peux pas trahir le secret judiciaire : l'officier de police judiciaire n'est pas autorisé à divulguer les informations de ses auditions ». Vu son refus, je vais voir les policiers de garde. Contrairement au commandant, ceux-ci m'approchent et je leur explique la raison de ma présence dans leurs services. Ils sont contents que je sois là car ils peuvent bavarder avec un nouveau venu. Nous parlons de tout et de rien. Profitant de leur ouverture, je leur demande ce qu'ils font précisément aux Services spéciaux. Ils me répondent qu'ils doivent « assurer la sécurité des Services spéciaux et des détenus ».

De notre entretien, j'apprends qu'il y a huit cellules de détention : une cellule centrale à côté du corps de garde et sept autres dans une cour. Je leur demande si je peux voir les cellules, et ils n'y voient aucun inconvénient. Je constate que les cellules de détention sont très étroites et ne peuvent abriter plus de quatre détenus, à moins que certains ne restent debout. Cinq de ces cellules sont opérationnelles et ont des portes. A l'intérieur, il n'y a ni lit, ni matelas, ni électricité. Les conditions hygiéniques sont absolument déplorables. De ce contact avec les policiers de garde, $j$ 'acquiers une première vue des infrastructures des Services spéciaux.

La même nuit, je sollicite la collaboration d'un autre commandant. J'essaye de paraître plus convivial, détendu et coopératif. Le climat de méfiance s'estompe rapidement et je suis installé par ce commandant dans son bureau.

\section{Samedi : l'étudiant finaliste}

Ce samedi, je travaille de jour. Vers 14 heures, le commandant qui m'avait repoussé la veille au soir, me voyant auprès de son collègue, commence à s'intéresser à moi. Il 
me suit dans le bureau et me demande de lui réexpliquer le but de ma présence dans le service et ce, en présence de son collègue. Ce dernier, qui me prend pour un stagiaire, répond à ma place. Comme pour confirmer ce qu'il dit, il me confie un tas de documents à classer comme tâche journalière. Pour moi, c'est une belle ouverture, car en classant ces documents, je peux observer de quelle manière on traite les plaintes : je remarque rapidement que le commandant possède deux registres parallèles et qu'il perçoit de l'argent des plaignants ou des prévenus qui cherchent ainsi à infléchir ses décisions.

Avant qu'il ne parte, je lui précise à nouveau le motif de ma présence dans le service : je ne suis pas un stagiaire mais bien un chercheur. Il me considérait en effet comme un étudiant finaliste qui mène ses recherches pour son mémoire. Malgré mes précisions, il continue à m'associer soit à un stagiaire soit à un étudiant finaliste. Lubumbashi étant une ville universitaire, les habitants sont habitués aux étudiants qui mènent des enquêtes auprès d'eux et ont tendance à projeter ce statut sur tout chercheur de terrain. Ce statut, quoique inexact, m'a permis de bénéficier d'une relative confiance pour la suite de mes enquêtes.

Le même jour, le commandant avec qui je travaille est relayé par un autre. Le nouvel officier de police judiciaire se montre très méfiant envers moi et ne répond à mes questions que par un hochement de tête. Je décide alors de changer d'approche : je ne lui parle plus d'enquêtes mais d'autres sujets de la vie courante (le football, la politique, etc.), sujets qui vraisemblablement captivent cet homme. Ce jour-là, nous nous séparons chaleureusement.

\section{Dimanche : au corps de garde}

Je le retrouve le dimanche 4 août, alors que je travaille de nuit pour la seconde fois. Il me salue fort amicalement et me demande si j'ai suivi les informations à la radio. Il se met à commenter des nouvelles sur la politique au Congo et je discute avec lui sans reparler de l'enquête. Après cette conversation, il prend le registre des plaintes et se met à m'en expliquer le contenu. Je lui pose alors des questions auxquelles il me répond très directement : il sera sans doute mon meilleur informateur au cours de cette enquête.

Je constate cependant que l'ambiance de la nuit est très différente de celle de la journée. La nuit est un autre monde : le commissariat vit replié, la hiérarchie s'estompe. Pendant la journée, les commandants sortent constamment et vont parfois auditionner les plaignants et prévenus dans un autre bureau pour se soustraire à mes observations, ce qui est impossible de nuit. Durant la nuit, la convivialité s'installe car on est appelé à veiller ensemble. Les policiers et moi dialoguons, et nous nous racontons des blagues pour passer le temps car la vie est ralentie, alors que pendant la journée, tous s'affairent à leurs tâches.

Alors que j'assure ma permanence dans le bureau d'investigation auquel je suis attaché, je constate vers 21 heures qu'il n'y a plus aucun plaignant. L'OPJ, pris de sommeil, s'endort tout doucement. Je décide de rejoindre le groupe de policiers chargés de la sécurité des détenus avec qui j'avais eu des contacts le vendredi.

Je les trouve en train de préparer le café. Je me comporte familièrement avec eux et leur remets de l'argent pour qu'ils achètent du pain. Ils me proposent de prendre place à leur table. Ils me posent quelques questions sur la vie au campus, car tous, malgré mes explications, me prennent encore pour un stagiaire en droit. Subitement, l'un d'eux se met à me parler de leurs rudes conditions de travail. Un peu plus loin, une prisonnière grelotte de froid à la belle étoile. Je leur demande pourquoi elle n'est pas dans une cellule. Ils me 
répondent qu'elle a mérité une punition (eko na mériter punition). D'ailleurs, elle doit entrer dans la cellule buluo. Je leur demande de quoi il s'agit. L'un d'eux m'explique que c'est une cellule où l'on met les détenus « têtus ». Dans cette cellule, ils ne peuvent rester plus de quinze minutes sans supplier d'être tirés de là, parce qu'il y a des punaises, des poux et des puces. Je joue à l'incrédule pour les inciter à me faire visiter la cellule. L'un d'eux m'y conduit. Le seuil de la porte à peine franchi, je remarque que les murs sont tâchés du sang de ces insectes écrasés. Je suis consterné.

Constatant que les policiers sont communicatifs, j'en profite pour poser quelques questions et j'apprends ainsi beaucoup de choses jusqu'alors dissimulées. Dans l'ambiance du partage de café, les policiers m'avouent qu'ils fouettent les détenus tôt le matin pour ne pas alerter les gens. Questionnés sur la raison de cette pratique, ils répondent vaguement que cela vise à les discipliner. Cette réponse ne me convainc pas. Vers minuit, je m'arrange avec le policier qui m'avait fait visiter les cellules. Je lui repose alors la question sur la maltraitance des détenus : il me révèle que c'est une façon de gagner un peu plus d'argent. Les détenus qui sont fouettés sont ceux qui n'ont rien donné à la ronde matinale réalisée par les policiers de garde. Ceux qui donnent 100 francs $(0,25 €)$ sont épargnés. C'est l'une des raisons pour laquelle les détenus demandent de l'argent à leurs visiteurs. Bref, cette maltraitance systématique est utilisée par les policiers pour ramener quelque chose à la maison.

Formellement, les policiers attachés au poste ne peuvent le quitter sans une autorisation expresse de la hiérarchie. Toutefois vers minuit, le groupe des policiers se scinde en deux. Cinq d'entre eux partent traquer les prostituées et les ivrognes pendant que les deux autres restent avec moi afin d'assurer la permanence. Quand leurs compagnons reviennent avec des prostituées, ceux qui sont restés s'improvisent en chefs pour la circonstance. Leurs compagnons présentent le cas des prévenus en aggravant la situation et les infractions. Le chef improvisé tranche toujours en défaveur des prévenus, sans leur laisser l'opportunité de s'exprimer. Cela aboutit toujours à l'incarcération des prévenus, sans qu'aucune trace administrative ne subsiste.

J'assiste à ces scènes avec passivité : mon rôle est d'observer. J'esquisse un sourire afin de mettre les agents en confiance pour qu'ils agissent comme à leur habitude. Je n'écris pas, j'observe. Après la conclusion de cette rafle, nous commentons la scène. Je plaisante en imitant le comportement des prévenus et les prises de décision du « chef ». Ils rient et les commentaires vont bon train. L'un d'eux me dit alors : « chef", si on ne fait pas ça, les enfants n'auront rien à manger. Chacun dans son secteur monte des stratégies pour avoir de l'argent. D'ailleurs, il te faut aussi un peu d'argent pour le transport : c'est le droit de l'œil ». Je ris et je lui réponds : « tu as raison, mais ça viendra ». En effet, quand on partage de l'argent devant une personne, cette dernière peut réclamer également son dû pour avoir assisté au partage : c'est le « droit de l'œil ».

J'ai vécu une scène semblable le mercredi 7 août. On amène aux Services spéciaux une jeune prostituée de dix-neuf ans qui faisait l'amour sous un arbre. Son client s'est échappé. Les policiers rapportent le fait. L'infraction d'attentat à la pudeur est retenue contre la fille qui est astreinte à passer une nuit au cachot avant que son dossier ne soit transféré au Parquet. La prostituée supplie le commandant de circonstance qui exige

2. C'est un statut que l'on attribue aux étudiants. Dans la perception populaire, l'étudiant est appelé un jour à assumer des grandes responsabilités. 
3000 francs pour classer le dossier. La fille ne possède sur elle que 500 francs que le chef empoche. Elle ne va pas au cachot mais dans une pièce à l'intérieur des services de garde. Un policier me souffle que l'on va la « coucher » avant de la relâcher.

Ces réalités, je ne pouvais jamais les vivre pendant la journée.

\section{Lundi : les registres}

«L'observation participante peut prendre une forme plus active ou plus passive selon le niveau d'implication de l'observateur relativement aux événements et aux points de vue des sujets » (Lessard-Hebert et al. 1997 : 102). Les deux premiers jours de l'enquête, ma participation était passive. La confiance aidant, je suis amené pour la première fois, ce lundi 5 août, à dresser des procès-verbaux ou à verbaliser les dossiers. A chaque fois que le commandant reçoit une plainte, il me la renvoie ou m'associe à l'audition. Le plus souvent, je suis transformé en « officier de police judiciaire faisant fonction ». Bien que je sois néophyte en la matière, je ne peux renoncer à la proposition. A la fin de l'audition, une discussion s'ensuit et me permet de cerner certaines réalités.

Je travaille sur base de trois registres, à savoir, le registre des plaintes (celles que la population vient déposer au commissariat ou au poste de police), le registre de permanence (où l'on consigne tous les événements survenus pendant les permanences des commandants qui se relayent), enfin le cahier d'écrou (qui donne la situation des détenus dans les cellules). Certains commandants ne veulent pas que je puisse consulter le registre de plaintes, avançant que je ne suis pas assermenté. Pour m'en empêcher, certains commandants enferment les registres de plaintes dans leurs tiroirs ou les emportent dès qu'ils sortent des bureaux.

Pour contourner cette difficulté, j'enregistre toutes les plaintes survenues en ma présence. Par la suite, quand j'ai pu accéder aux registres des plaintes, il s'est avéré que les plaintes monnayées ne sont pas mentionnées dans les registres. Durant la période des enquêtes, j'ai enregistré 64 plaintes alors que pour la même période, le registre des plaintes ainsi que celui de permanence n'en font apparaître que treize : seules les plaintes mineures sont notées (coups et blessures, vol simple, injures publiques, conflits de bail, etc.). Paradoxalement donc, l'intérêt des registres est à chercher dans la non inscription des plaintes, laquelle apprend beaucoup sur les pratiques des commissariats.

\section{Mardi : le garagiste incarcéré}

Formellement, lorsque les plaintes arrivent aux Services spéciaux, ce sont les officiers de police judiciaire qui instruisent le dossier, fixent les amendes et font le rapport au commandant titulaire et/ou à son second. Ces derniers déterminent l'issue du problème qui leur est soumis en classant sans suite, en renvoyant devant des instances supérieures ou en négociant des amendes transactionnelles dont les montants s'échelonnent légalement de 20USD à 1 000USD. Pour les cas observés lors de ma permanence, le taux des amendes varie d'une personne à l'autre, selon l'appréciation des commandants.

Ce mardi 6 août, vers dix heures alors que j'assiste à l'audition d'un cas mineur de soupçon de vol, je vois entrer un plaignant. Le propriétaire d'une épave de voiture Peugeot 504 détournée par un garagiste vient porter plainte contre ce dernier à qui il avait confié la garde de son bien. Avant de déposer la plainte, le plaignant glisse au commandant une liasse des billets de 100 francs, puis déclare : « je ne veux pas discuter avec le garagiste, il m'a tellement fait de mal que je ne veux même pas le rencontrer. L'argent que je te donne... dès qu'il arrive, tu le fais fouetter et tu le jettes en prison ». Le commandant 
perçoit l'argent et établit un mandat d'amener qu'il confie aux policiers. Je demande au commandant pourquoi un mandat d'amener au lieu d'une convocation ? Il me répond que c'est une grande affaire qu'il faut terminer rapidement.

Quelques minutes plus tard, le garagiste entre, escorté et menottes aux poignets. Le commandant prend à peine le temps de lui demander son nom avant de le jeter en prison. Une heure après, il le rappelle et le garagiste s'explique. Le commandant le condamne et fixe des amendes à 50USD après quoi il devra, dit-il, transférer le dossier au parquet. La famille du garagiste, arrivée sur les lieux, supplie le commandant de ne pas transférer l'affaire au parquet : le garagiste serait d'accord de fournir une autre épave et de payer 30USD. Avant que le plaignant ne soit de retour, la femme du garagiste glisse 30USD dans la main du commandant. Ce dernier rassure la femme et le prévenu en disant qu'il n'enverra pas le dossier au parquet et tentera de persuader le plaignant de clôturer l'affaire.

Quelques heures plus tard, le plaignant est de retour avec un air dédaigneux. Le commandant confronte les deux antagonistes : pour satisfaire le plaignant, il exige qu'une autre épave lui soit donnée et que le prévenu soit incarcéré. Le garagiste déclare avoir vendu l'épave et s'engage à en donner une autre à la place, puis est remis au cachot. Le plaignant est très satisfait : en guise de remerciement, il donne 25USD au commandant. Ce dernier lui demande d'être là le lendemain à 9 heures pour retirer l'épave.

Une demi-heure après que le plaignant soit parti, le commandant libère le garagiste et lui intime l'ordre d'être au bureau au plus tard à sept heures : passé ce délai, il sera pris pour un fuyard. En effet, il s'agissait de conforter l'illusion du plaignant que le garagiste avait bien passé la nuit au cachot...

J'assiste passivement à ces transactions, réalisant que pour cette seule affaire, le commandant a empoché 65USD : il ne fait plus preuve d'aucune retenue devant moi. Si le commandant s'est taillé la part du lion, les policiers qui ont escorté le garagiste ont à leur niveau soutiré quelques francs congolais. C'est un droit qui leur est reconnu et qu'on appelle mbongo ya makolo (l'argent pour les pieds). Je comprends de plus en plus clairement que la justice n'est que de façade dans les Services spéciaux : plaignants et prévenus rivalisent à coups de pots-de-vin.

\section{Mercredi : « Prends cet argent!"»}

L'avant-dernier jour, le 7 août, un commandant très confiant me met dans une situation fort embarrassante en me confiant l'audition d'un cas de vol. Quand le plaignant entre au bureau, le commandant le dirige vers moi parce qu'il verbalise un autre cas. Avant de poser son problème, le plaignant sort une liasse de billets de 100 francs et me la tend en me disant qu'il souhaite que le prévenu soit copieusement tabassé avant la confrontation. Je refuse poliment. Le plaignant insiste. Comme je reste sur mes positions, le commandant intervient en me disant «Prends cet argent, ce n'est pas de la corruption. Celui qui te le donne sait que tu ne le lui as pas demandé ». Je refuse avec politesse et le commandant prend l'argent en mon nom. Après le départ du plaignant, le commandant manifeste son mécontentement envers ma réaction. Je dois m'expliquer : je dis que ma conviction religieuse m'interdit la corruption. Or, le plaignant m'avait tendu l'argent pour que je fasse mal au prévenu avant même de l'entendre. Je ne pouvais pas cautionner cette injustice. Le commandant refuse pour sa part de considérer ce geste comme de la corruption. Je pense qu'il voulait que je prenne cet argent pour s'assurer vraiment que je suis de leur côté. Pour le calmer, je détourne la conversation en lui demandant pourquoi 
les plaignants donnent de l'argent sans qu'on le leur demande. Il répond que c'est pour eux une façon d'infléchir la décision du commandant. Nous commentons la situation et je défends ironiquement sa cause en disant que s'il y a corruption, ce sont les plaignants qui l'imposent : en effet, je n'avais rien demandé mais on a voulu me donner ! Le commandant rit et l'affaire est clôturée. Depuis ce jour, il m'appelle «pasteur»!

\section{Conclusion}

Les enquêtes menées aux services spéciaux ont été délicates. La difficulté résidait dans le fait que les policiers développaient dès le premier contact une méfiance, une peur que leurs propos soient utilisés contre eux, ou que je découvre et dénonce leurs irrégularités auprès de la hiérarchie. La confiance a pu être acquise au fil des contacts. Le premier jour a été marqué par le refus et la méfiance absolus. Ceux-ci cédèrent progressivement place à la collaboration. Dès le deuxième jour, j'étais installé dans un bureau où j'avais l'opportunité d'écouter, d'observer et d'écrire les faits qui m'intéressaient. Le troisième jour, l'intégration était manifeste en ce sens que les policiers avec qui j'avais eu des contacts préliminaires pouvaient me révéler des aspects secrets de leur mode de fonctionnement, comme il est apparu lors de la veillée du samedi. Et au sixième jour, un commandant me considérant presque comme l'un des leurs ne s'est pas gêné pour m'introduire dans leur économie parallèle. Les contacts permanents font tomber les obstacles à l'enquête : la familiarité se consolide au quotidien. C'est l'avantage de l'enquête intensive : on ne peut tout cacher continuellement.

Assuré de la confiance auprès des policiers, j'ai recouru surtout à l'observation pour recueillir mes informations, y compris dans les domaines apparemment inaccessibles. Cette observation doit suivre un enchaînement de trois savoir-faire fortement imbriqués : percevoir, mémoriser, noter. «Elle suppose un va-et-vient permanent entre les perceptions, leur explication mentale, leur mémorisation et le cahier sur lequel l'enquêteur prend des notes. Elle est une vigilance aiguisée par des informations extérieures et des questions qui évoluent au fur et à mesure de la poursuite du travail » (Beaud et Weber 1998 : 143).

Ma participation à certaines activités fut très riche d'enseignements : l'audition des plaintes m'a facilité la compréhension du processus de corruption; la pause-café avec les policiers de garde m'a permis d'apprendre beaucoup sur les sévices pratiqués. Si j'avais dû me limiter à la lecture des registres et aux entretiens formels, je n'aurais jamais rien pu apprendre de tout cela.

\section{Références bibliographiques}

Beaud, Stéphane et Florence Weber, 1998. Guide de l'enquête de terrain. Produire et analyser des données ethnographiques. Paris : La Découverte.

Lessard-Hebert, Michelle, Gabriel Goyette et Gérald Boutin, 1997. La recherche qualitative : fondements et pratiques. Bruxelles : De Boeck Université.

Kaumba Lufunda (éd.), 2004. Approche de la criminalité à Lubumbashi. Lubumbashi : rapport de recherche de l'Observatoire du changement urbain.

Poupart, J., 1997. «L'entretien de type qualitatif : considérations épistémologiques, théoriques et méthodologiques », in Gaëtan MoRIn (éd.), La recherche qualitative. Enjeux épistémologiques et méthodologiques, pp. 173-209. Québec : Bibliothèque nationale du Québec. 